\title{
Bone in the breast: Clinical, radiological and pathological correlation
}

An 84-year-old woman presented with a left breast mass that had been rapidly growing over a few months. On clinical examination, there was a large and firm left breast mass with no overlying skin change. The right breast was normal. The patient was treated in accordance with the Declaration of Helsinki, and Institutional Review Board approval was obtained.

The patient was evaluated with mammography (Fig. 1) and ultrasound scan of the left breast (Fig. 2).

Which is the most likely diagnosis?
A. Invasive ductal carcinoma
B. Invasive medullary carcinoma
C. Involuting giant fibroadenoma
D. Phyllodes tumour with heterologous osteoid component
E. Fat necrosis

Findings and diagnosis. Mammography showed a large, dense, left breast mass with lobulated, circumscribed margins. It contained a cluster of almost bone-like coarse calcifications (Fig. 1). Sonography demonstrated a $13 \mathrm{~cm}$, lobulated, complex solid cystic mass at the left 10 to 3 o'clock position with ill-defined margins (Fig. 2).

No axillary or distant metastases were detected on staging computed tomography.

Ultrasound-guided core needle biopsy through the solid component was performed. Microscopic examination revealed leaf-like stromal fronds with a spindle cell proliferation suggestive of a phyllodes tumour (PT) (Fig. 3). There was microscopic ossification, but no abnormal stromal cells with significant atypia were seen associated with the bone, thus favouring a benign osseous metaplastic process. Excision was advised to exclude underlying malignancy of the phyllodes tumour. In view of its large size and risk of inadequate margins, the patient underwent a mastectomy.

Pathology of the mastectomy specimen showed a malignant PT with a heterologous osteosarcomatous component (Fig. 4).

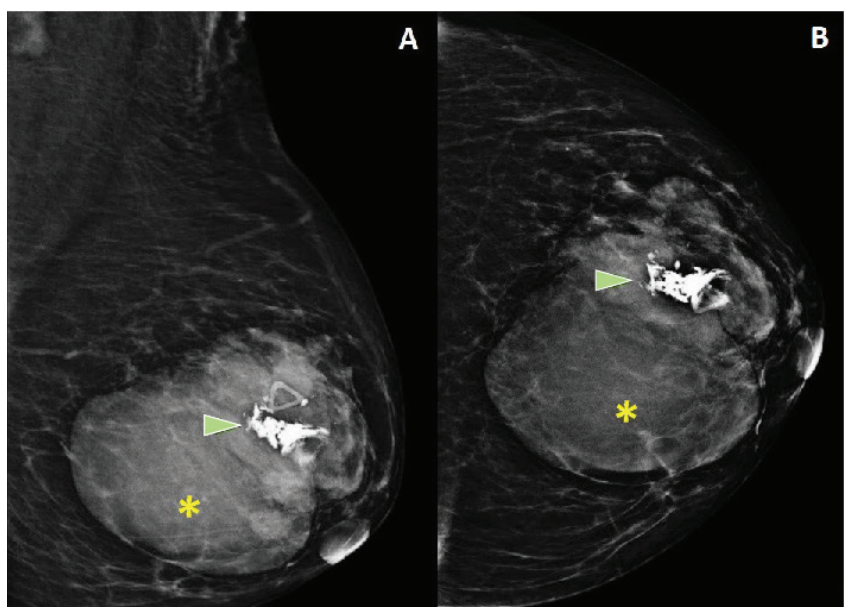

Fig. 1. Mammogram of the left breast (A) mediolateral oblique and (B) craniocaudal views show a lobulated mass (asterisks) occupying most of the breast. No spiculations are seen. The mass contains a prominent cluster of almost bone-like coarse calcifications, which appear very thick, haphazard in pattern and sharply angulated (arrowheads).

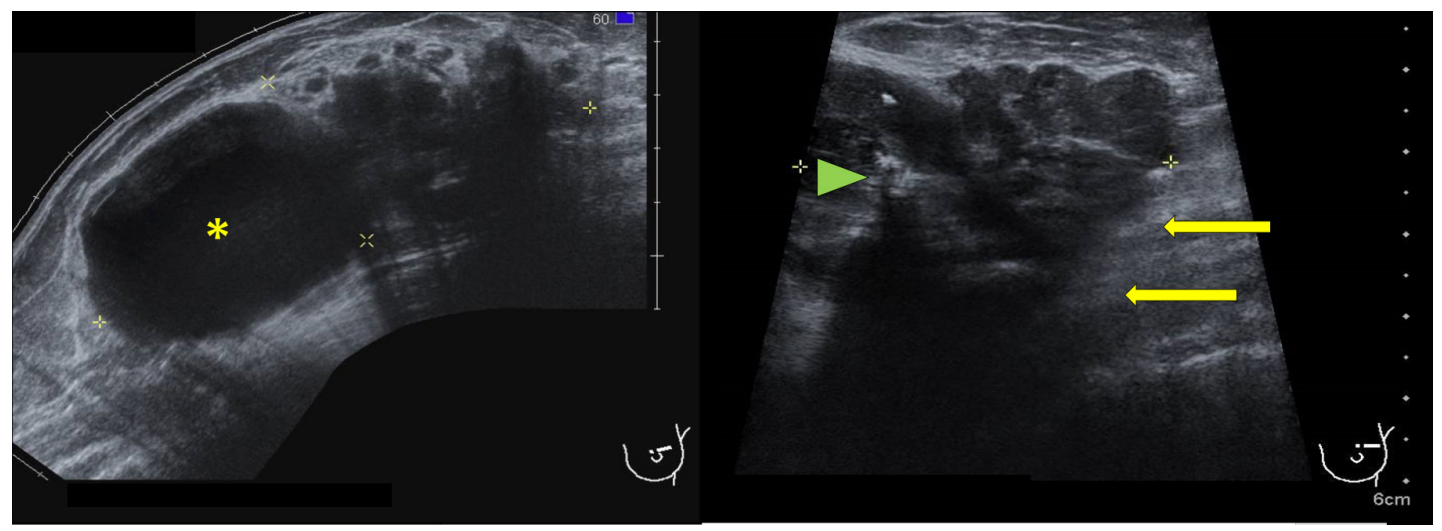

Fig. 2. Ultrasound of the left breast shows a $13 \mathrm{~cm}$ solid-cystic mass. There is an eccentrically located cystic component with circumscribed margins (asterisk), and an adjacent lobulated hypoechoic solid component with partially indistinct margins and posterior acoustic shadowing (long arrows). Internal echogenic foci with posterior acoustic shadowing (arrow head) correspond with the coarse calcifications on mammography. 
Discussion. In an elderly patient presenting with a rapidly enlarging breast mass, the important diagnostic differential considerations will be phyllodes tumour, primary breast sarcomas, medullary carcinomas and any type of high-grade invasive cancers.

The majority of invasive ductal carcinomas (IDCs) present as a mass with spiculated margins, which was not a feature in this case. IDC masses may contain calcifications, which are often associated with a ductal carcinoma in situ (DCIS) component. The calcifications of DCIS are usually pleomorphic or linear branching type in morphology and are $<0.5 \mathrm{~mm}$ in size. ${ }^{1}$ These coarse calcifications seen in this case are not typical for IDC.

Medullary carcinomas are known to be rapidly growing and can present as circumscribed lobulated masses. However, they are not typically associated with calcifications, hence this diagnosis is unlikely. ${ }^{1}$

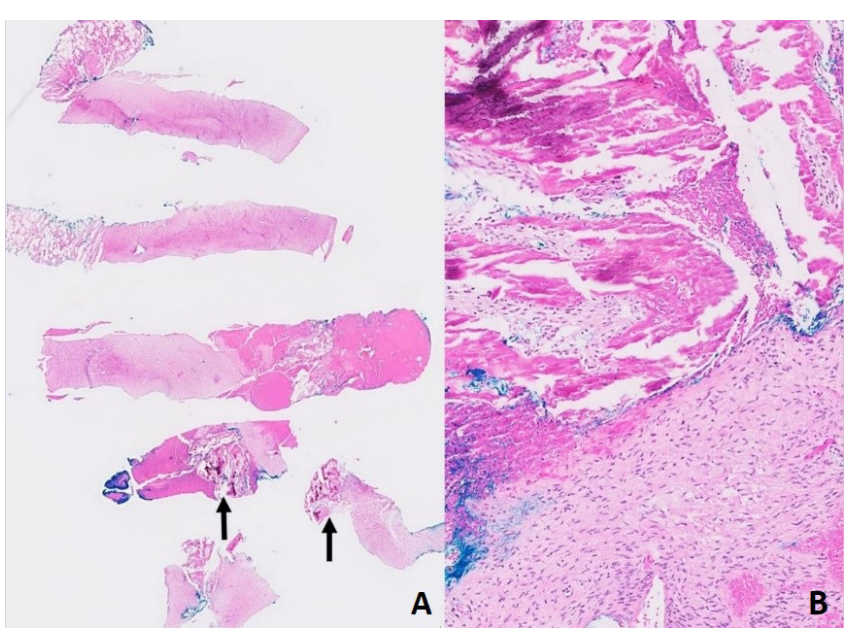

Fig. 3. (A) Core needle biopsy of the breast mass shows a spindle cell lesion with areas of ossification (arrows). (B) High magnification of the ossified portion shows association with spindle cells with relatively bland cytologic features.
Fat necrosis, which is typically associated with trauma or intervention, has a variable imaging appearance. It can develop as an irregular, spiculated mass or demonstrate clustered microcalcifications, and overlap with the imaging appearance of malignancy. However, the mass usually contains fatty radiolucent areas, and the calcifications are characteristically peripheral at the rim. Its sonographic appearance is non-specific. It can appear as an anechoic cyst, solid mass or a complex solid-cystic lesion, and should be correlated with the mammogram findings. ${ }^{2}$ In this case, the dense solid component lack fatty radiolucent elements and the calcifications are unlike those associated with fat necrosis, which are usually dystrophic or rimmed in appearance with lucent centres.

Fibroadenomas and PT are both fibroepithelial lesions, and have overlapping radiological and histological features. However, giant fibroadenomas $\geq 5 \mathrm{~cm}$ in size are seen in younger premenopausal women and do not grow after menopause. On imaging, fibroadenomas tend to be smaller $(<4 \mathrm{~cm})$ and are usually wider than tall in orientation. Both lesions can have lobulated margins but PT tends to have bulging borders. ${ }^{3}$ Involuting fibroadenomas can also demonstrate coarse, popcorn-like calcifications that are $>2 \mathrm{~mm}$ in size. These calcifications typically show smooth margins, unlike the irregular and angulated margins seen in this case. Intra-tumoural cystic components are usually due to clefts between the bulging nodular protuberances, and are more commonly seen in PT compared to fibroadenomas. Cystic infarctions in rapidly growing fibroadenomas and PT may also give rise to cystic areas. ${ }^{3}$

PTs are rare fibroepithelial neoplasms that account for $<1 \%$ of all breast neoplasms, usually affecting women $40-50$ years of age. ${ }^{4}$ Based on the histological characteristics, which include nature of the tumour

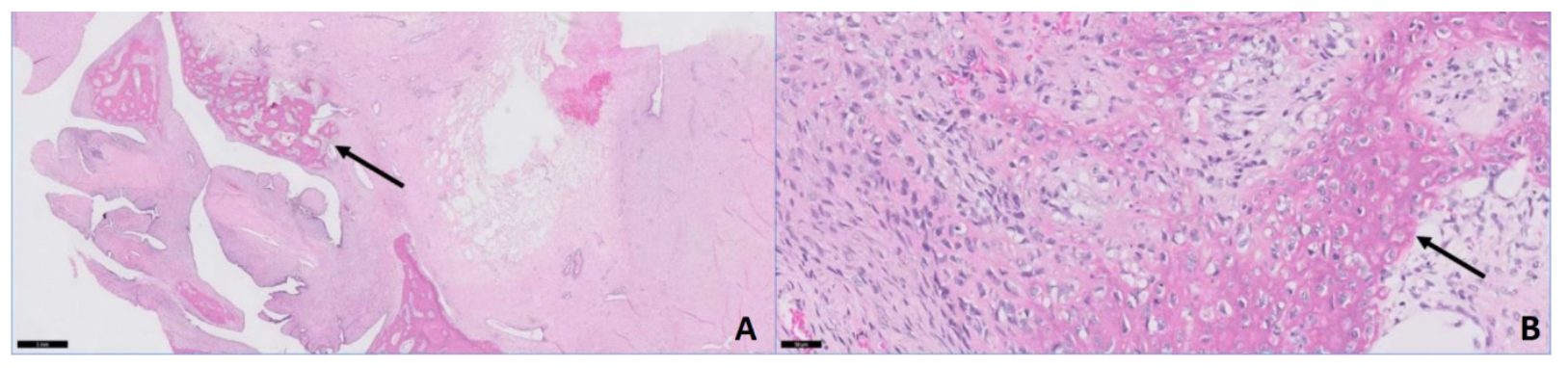

Fig. 4. (A) Histopathology of the tumour in the resected mastectomy specimen at low magnification shows characteristic stromal fronds from the phyllodes tumour. It contains haphazard bony trabeculae (arrow), which correspond with the calcific pattern seen on mammography. (B) High magnification shows anastomosing pink osteoid (arrow) among abnormal cells with discernible mitoses, consistent with malignant osteoid production in osteosarcoma. 
borders, degree of stromal cellularity, stromal overgrowth, cellular atypia and the number of mitoses per 10 high power fields, they are classified into benign, borderline and malignant categories.

A study found that phyllodes tumour cells have properties of mesenchymal stem cells. ${ }^{5}$ As malignant phyllodes tumours can have heterologous differentiation along various mesenchymal lineages (bone, cartilage, fat, muscle, etc.), the authors hypothesised that phyllodes tumour cells have mesenchymal stem cell properties and indeed, some similarities were found. ${ }^{5}$ This ability to differentiate into various mesenchymal lineages and in this case, transform into an osteosarcomatous component with bone formation is a known feature of malignant phyllodes tumours.

PT commonly presents as a large, rapidly growing mass. Features of a regular shape with generally circumscribed margins that lack spiculations help differentiate it from other invasive breast malignancies. ${ }^{6}$ The margins of PT also tend to be bulging and lobulated due to stromal protuberances. ${ }^{6}$ Ill-defined margins and intra-tumoural cystic spaces, as demonstrated in this case, favour malignancy. Although coarse calcifications have been reported in PT, they are rare due to rapid tumour growth. ${ }^{7}$

Coarse calcifications may be seen in an involuting fibroadenoma-the calcifications of calcified fibroadenomata are typically smoothly marginated and popcorn-like. ${ }^{8}$ This is different from the coarse calcifications in this case, which had angulated margins.

Other imaging differentials include primary breast sarcomas and metaplastic breast carcinoma. Primary breast sarcomas are a heterogeneous group of mesenchymal tumours without epithelial components, which include angiosarcoma, osteogenic sarcoma, fibrosarcoma, liposarcoma and malignant fibrous histiocytoma. ${ }^{9}$ Metaplastic carcinoma is an invasive carcinoma with mesenchymal and epithelial components, and shows coexistence of matrix-producing, spindle cell, sarcomatous or squamous differentiations. Both types of tumours can present as a large rapidly growing mass of regular shape with circumscribed, lobulated margins. ${ }^{10-12}$ They may appear as solid-cystic masses on sonography, ${ }^{10,13}$ and may have coarse calcifications if there is osseous heterologous differentiation, similar to the case presented. Metaplastic breast carcinomas tend to be associated with stromal distortion. ${ }^{14}$ Both types of tumours are rare, accounting for $<1 \%$ of all breast cancers; phyllodes tumours are relatively more common in comparison.

On microscopy, malignant osteoid formation can be mistaken as collagen, especially when the osteoid is not calcified. Also, if histological ossification is not accompanied by malignant cells, interpretation as a benign metaplastic process is likely. There may be undersampling with core needle biopsies, further contributing to the challenges of accurate recognition. Knowledge of the radiological features can help alert the pathologist to the possible malignant nature of the tumour. Presurgical diagnosis of a malignant PT will be useful for surgical planning, especially if a wide local excision is planned. Surgery should aim to achieve negative margins for malignant PT to reduce the risk of local recurrence. Wide local excision with $10 \mathrm{~mm}$ margins, without axillary staging, is the current recommended treatment according to the recent US National Cancer Comprehensive Network guidelines. ${ }^{15}$

\section{REFERENCES}

1. Berg WA, Birdwell RL, Gombos E, et al. Diagnostic Imaging: Breast. 1st Ed. Salt Lake City: Amirsys; 2006.

2. Taboada JL, Stephens TW, Krishnamurthy S, et al. The many faces of fat necrosis in the breast. AJR Am J Roentgenol 2009; 192:815-25.

3. Gatta G, Iaselli F, Parlato V, et al. Differential diagnosis between fibroadenoma, giant fibroadenoma and phyllodes tumour: sonographic features and core needle biopsy. Radiol Med 2011; 116:905-18.

4. WHO Classification of Tumours. Breast tumours. WHO classification of tumours series, 5th Ed, Vol 2. Lyon (France): International Agency for Research on Cancer; 2019.

5. Lin JJ, Huang CS, Yu J, et al. Malignant phyllodes tumors display mesenchymal stem cell features and aldehyde dehydrogenase/ disialoganglioside identify their tumor stem cells. Breast Cancer Res 2014;16:R29.

6. Liberman L, Bonaccio E, Hamele-Bena D, et al. Benign and malignant phyllodes tumors: Mammographic and sonographic findings. Radiology 1996;198:121-4.

7. Chao TC, Lo YF, Chen SC, et al. Sonographic features of phyllodes tumors of the breast. Ultrasound Obstet Gynecol 2002;20:64-71.

8. Bennett DL, Merenda G, Schnepp S, et al. Primary breast osteosarcoma mimicking calcified fibroadenoma on screening digital breast tomosynthesis mammogram. Radiol Case Reports 2017; 12:648-52.

9. Matsumoto RAEK, Hsieh SJK, Chala LF, et al. Sarcomas of the breast: Findings on mammography, ultrasound and magnetic resonance imaging. Radiol Bras 2018;51:401-6. 
10. Smith TB, Gilcrease MZ, Santiago L, et al. Imaging features of primary breast sarcoma. AJR Am J Roentgenol 2012;198:W386-93.

11. Dekkers IA, Cleven A, Lamb HJ, et al. Primary osteosarcoma of the breast. Radiographics 2019;39:626-9.

12. Yang WT, Hennessy B, Broglio K, et al. Imaging differences in metaplastic and invasive ductal carcinomas of the breast. AJR Am J Roentgenol 2007;189:1288-93.

13. Günhan-Bilgen Isil, Memiş A, Ustün EE, et al. Metaplastic carcinoma of the breast: Clinical, mammographic and sonographic findings with histopathologic correlation. AJR Am J Roentgenol 2002;178:1421-5.

14. Park JM, Han BK, Moon WK, et al. Metaplastic carcinoma of the breast: Mammographic and sonographic findings. J Clin Ultrasound 2000;28:179-86.

15. National Comprehensive Cancer Network. NCCN Clinical Practice Guidelines in Oncology: Breast cancer (Version 5.2020).
Cheryl Hui Shan Lim, ${ }_{F R C R}$, Chee Hao Lester Leong ${ }^{2}$ MMed, Sue Zann $\underline{\operatorname{Lim}}^{3}{ }_{F R C S}$, Myat Naing $\underline{\mathrm{Su}}^{4}{ }_{\text {MMed Sc (Radiology), }}$ Tammy Hui Lin Moey ${ }^{2}$ FRCR, Kwang Yong Timothy Tay ${ }^{5}$ FRCPath, Puay Hoon $\underline{\operatorname{Tan}}{ }^{5}$ FRCPA

${ }^{1}$ Department of Radiology, Sengkang General Hospital, Singapore ${ }^{2}$ Department of Diagnostic Radiology, Singapore General Hospital, Singapore ${ }^{3}$ Department of General Surgery, Singapore General Hospital, Singapore ${ }^{4}$ Imaging Department, Zabuthiri Specialist Hospital, Naypyitaw, Myanmar ${ }^{5}$ Division of Pathology, Singapore General Hospital, Singapore

Correspondence: Dr Cheryl Hui Shan Lim, Department of Radiology, Sengkang General Hospital, 110 Sengkang E Way, Singapore 544886. Email: cheryl.lim.h.s@singhealth.com.sg 